
\title{
R Research Square \\ Comparison of the Levels of Cardiac Troponin I in Patients with Duchenne and Becker Muscular Dystrophies to Assess Cardiac Dysfunction
}

Hiroshi Yamaguchi

Kobe University

Hiroyuki Awano ( $\square$ awahiro@med.kobe-u.ac.jp )

Kobe University

Tetsushi Yamamoto

Kobe University Hospital

Masafumi Matsuo

Kobe Gakuin University

Kazumoto lijima

Kobe University

\section{Research Article}

Keywords: alpha-actinin-3, cardiomyopathy, dystrophinopathy, heart failure, muscular dystrophy, echocardiography

Posted Date: February 3rd, 2021

DOl: https://doi.org/10.21203/rs.3.rs-154169/v1

License: (c) (1) This work is licensed under a Creative Commons Attribution 4.0 International License. Read Full License 


\section{Abstract}

Background: Cardiac troponin I (cTnl), uniquely expressed in the myocardium, is a marker for acute myocardial injury. Its clinical significance in Duchenne and Becker muscular dystrophy (DMD and BMD) and its relation to alpha-actinin-3 (ACTN3) genotype as a genetic modifier of cardiomyopathy are still unknown.

Methods and Results: Overall, 529 and 131 serum cTnl values of 127 DMD and 47 BMD patients, respectively, were reviewed. cTnl elevation was generally observed in the second decade of life. Both cTnI levels and the proportion of abnormal cTnl levels were significantly higher in DMD patients than in BMD patients (age range: $1<$ years $\leq 10$ and $10<$ years $\leq 18$ and $10<$ years $\leq 18$, respectively). Decreased left ventricular ejection fraction was observed after cTnl elevation in both populations. cTnl levels by age in DMD patients with ACTN3 null genotype tended to increase highly and early.

Conclusions: Myocardial injury indicated by cTnl was more common and severe in DMD patients than in BMD patients. cTnl elevation preceding cardiac dysfunction may represent an early phase of cardiomyopathy progression and may be a biomarker for early detection of cardiomyopathy in DMD and BMD patients. The ACTN3 null genotype may be a risk factor for early myocardial injury.

\section{Introduction}

Duchenne muscular dystrophy (DMD) is a lethal X-linked recessive muscular dystrophy caused by mutations in the $D M D$ gene, resulting in the absence of dystrophin $[1,2]$. Becker muscular dystrophy (BMD) is a milder allelic version of $D M D$, also caused by in-frame $D M D$ gene mutations that allow for the synthesis of shortened and partially functional dystrophin $[3,4]$. As dystrophin is expressed not only in skeletal but also in cardiac muscles, its absence or dysfunction affects the heart [5]. In fact, cardiomyopathy is common in patients with $\mathrm{DMD}$ or $\mathrm{BMD}[3,6]$. Long-term muscle deterioration, with significant implications for lung and heart dysfunction, is pathognomonic of DMD $[7,8]$. Recently, improved respiratory management has contributed to better respiratory status in patients with DMD; therefore, end-stage heart failure (HF) following cardiomyopathy is increasingly becoming the main cause of death in these patients [9]. However, physicians encounter difficulties in the early detection of cardiac dysfunction, especially in DMD, because of the early loss of ambulation, which reduces the load on the heart, thereby reducing cardiac symptoms $[10,11]$. Previous studies have shown that early diagnosis of cardiac involvement is important because timely initiation of cardioprotective medications relieves cardiac dysfunction and delays heart muscle remodeling $[12,13]$. Echocardiography is a primary and standard modality to screen for cardiac dysfunction in patients with muscular dystrophy [11]. However, it is not always adequate for detecting the early, clinically asymptomatic phase of disease progression because body habitus or scoliosis renders the acoustic imaging windows inadequate [14]. Cardiovascular magnetic resonance imaging (cMRI) is a new noninvasive imaging modality for detecting early cardiac dysfunction [15]. However, it is costly, not globally available, and requires sedation in young patients [11]. Despite the increased awareness of cardiac dysfunction in DMD and BMD patients, an 
average delay of 2.5 years between the onset of symptoms and the diagnosis of HF has been reported [16]. Therefore, accurate and low-cost biomarkers for early detection of cardiomyopathy are needed.

Cardiac troponin I (cTnl) is a member of the troponin complex and a major component of myofibrils [17]. cTnl is uniquely expressed in cardiac muscles [18], and because it appears in the blood following cardiac injury, it is used as a specific cardiac injury marker for acute myocardial infarction (AMI) in adults [19]. Although some studies have reported the utility of cTnl for evaluating cardiac function in muscular dystrophy, little is known about its characteristics and diagnostic value in these patients [18].

The alpha-actinin-3 (ACTN3) gene encodes alpha-actinin-3, one of the major structural components of sarcomeric Z-discs [20]. There is a common null variant-c.1729C > T (p.R577X) (rs1815739) (NM_001104.4) - of ACTN3 that results in the replacement of arginine $(\mathrm{R})$ with a premature stop codon $(\mathrm{X})$ at amino acid 577 in the muscle protein alpha-actinin-3, leading to the deficiency of alpha-actinin-3 in the individuals with the homozygous p.577X genotype [21]. We have previously reported that the ACTN3 homozygous p.577X genotype is associated with a lower left ventricular (LV) dilation-free survival rate in DMD [22], suggesting that ACTN3 is a genetic modifier of cardiomyopathy.

In this study, we compared cTnl levels in patients with DMD or BMD and assessed cTnl levels and cardiac function to determine whether $\mathrm{cTnl}$ is a biomarker of cardiac dysfunction in these patients. Furthermore, we evaluated the contribution of the ACTN3 genotype to the serum levels of cTnl.

\section{Results}

\section{Distribution of cTnl in patients with DMD and BMD by age}

A total of 174 patients (127 DMD and 47 BMD patients) were enrolled. For patients with DMD or BMD, 529 and 131 serum cTnl values were collected, respectively. All data on serum cTnl levels, including multiple measurements for each person by age, for patients with DMD or BMD are shown in Fig. 1a and $1 \mathrm{~b}$, respectively. During the first decade of life, serum cTnl levels were rarely elevated, and abnormal values were generally found in the second decade in both groups. The cTnl levels stabilized at a low level after the third decade in both groups. Markedly high values $(>2.0 \mathrm{ng} / \mathrm{mL})$ were observed in both patient groups during the second decade (Fig. 1a and b); these values were obtained from three DMD and two BMD patients. The three DMD patients who showed markedly high values had deletions of exons $56-62$, small insertions in exon 8 (c.783dupT), and small deletion in exon 18 (c.2230_2231delAG). The mutations in the two BMD patients were small deletions in exon 27 (c.3613delG) [27] and deep intron mutation in intron 4 (c.265-463A > G). There was no specific predisposition to any mutation position or type. None of the patients showed symptoms such as chest pain or electrocardiogram (ECG) findings related to $\mathrm{AMI}$ at the time of $\mathrm{cTnl}$ measurement.

Comparison of serum cTnl levels and proportions of abnormal cTnl levels between DMD patients and BMD patients 
To assess whether serum cTnl level or distribution differed between DMD and BMD, the highest serum cTnl value for each patient (only one value per patient over a lifetime) was adopted for statistical analysis. Thus, 127 and 47 values were collected for patients with DMD and BMD, respectively. Serum cTnl values and the proportions of patients with abnormal cTnl values were compared. The background data of the DMD $(n=127)$ and BMD $(n=47)$ patients at the time of serum cTnl assay are displayed in Table 1. There was no statistical difference in age between the DMD and BMD groups. Angiotensinconverting enzyme inhibitors, beta-blockers, steroids, non-invasive positive pressure ventilation were more commonly used in DMD patients. The comparison of the highest value of serum cTnl for each patient between DMD and BMD groups is shown in Table 2. The median (IQR) serum cTnl level in the DMD group was $0.06 \mathrm{ng} / \mathrm{mL}(0,0.16)$, which was higher than that in the BMD group $(0.01 \mathrm{ng} / \mathrm{mL}[0,0.05])$. When the data were divided into three patient age groups ( $1<$ years $\leq 10,10<$ years $\leq 18$, and $18<$ years), the median serum cTnl $(1<$ years $\leq 10,10<$ years $\leq 18)$ was statistically higher in patients with DMD than in those with BMD. In contrast, in patients $>18$ years, there was no statistical difference. The proportion of patients with abnormal serum cTnl levels was statistically larger in patients with DMD within the age range of $10<$ years $\leq 18$ than in BMD patients. 
Table 1

Patients' background characteristics

\begin{tabular}{|c|c|c|c|}
\hline & DMD $(n=127)$ & $\mathrm{BMD}(\mathrm{n}=47)$ & P-value \\
\hline Age, median (IQR), years & $13(8,18)$ & $15(10,18)$ & 0.19 \\
\hline \multicolumn{4}{|l|}{ Medication, n (\%) } \\
\hline ACEI & $42(33.1)$ & $7(14.9)$ & $0.022^{\$}$ \\
\hline ARB & $3(2.4)$ & $0(0)$ & 0.56 \\
\hline beta-blocker & $42(33.1)$ & $5(10.6)$ & $0.004^{\$}$ \\
\hline diuretic & $3(2.4)$ & $0(0)$ & 0.56 \\
\hline Steroid & $23(18.1)$ & $0(0)$ & $0.0006^{\$}$ \\
\hline \multicolumn{4}{|l|}{ Motor, n (\%) } \\
\hline Gait & $42(33.1)$ & $46(97.9)$ & $<0.0001^{\$}$ \\
\hline Walker & $1(0.8)$ & $0(0)$ & 1.0000 \\
\hline wheelchair & $84(66.1)$ & $1(2.1)$ & $<0.0001^{\$}$ \\
\hline \multicolumn{4}{|c|}{ Respiratory management, n (\%) } \\
\hline BiPAP or NPPV & $13(10.2)$ & $0(0)$ & $0.021^{\$}$ \\
\hline ventilator & $0(0)$ & $0(0)$ & - \\
\hline \multicolumn{4}{|c|}{$\begin{array}{l}\text { Abbreviations: ACEI, angiotensin-converting enzyme inhibitor; ARB, angiotensin II receptor blocker; } \\
\text { BiPAP, bi-level positive airway pressure; BMD, Becker muscular dystrophy; DMD, Duchenne muscula } \\
\text { dystrophy; NPPV, non-invasive positive pressure ventilation; IQR, interquartile range }\end{array}$} \\
\hline
\end{tabular}


Table 2

Comparison of serum cTnl levels and proportion of abnormal cTnl levels in DMD and BMD patients
$\operatorname{DMD}(n=127)$
$\operatorname{BMD}(n=47)$
P-value

cTnl, median (IQR), ng/mL

\begin{tabular}{llll} 
Total & $0.06(0.00,0.16)$ & $0.01(0.00,0.05)$ & $0.031^{*}$ \\
$(1<$ years $\leq 10)$ & $0.00(0.00,0.04)$ & $0.00(0.00,0.00)$ & $0.048^{*}$ \\
$(10<$ years $\leq 18)$ & $0.13(0.07,0.49)$ & $0.04(0.01,0.15)$ & $0.039^{*}$ \\
$(18<$ years $)$ & $0.03(0.00,0.08)$ & $0.02(0.01,0.04)$ & 0.74 \\
\hline Abnormal cTnl levels, $\mathrm{n} / \mathrm{n}(\%)$ & & & \\
Total & $71 / 127(55.9 \%)$ & $15 / 47(31.9 \%)$ & $0.006^{\$}$ \\
$(1<$ years $\leq 10)$ & $8 / 44(18.2 \%)$ & $1 / 14(7.1 \%)$ & 0.43 \\
$(10<$ years $\leq 18)$ & $49 / 57(86.0 \%)$ & $10 / 22(45.5 \%)$ & $0.0005^{\$}$ \\
$(18<$ years $)$ & $14 / 26(53.8 \%)$ & $4 / 11(36.4 \%)$ & 0.48
\end{tabular}

Abbreviations: BMD, Becker muscular dystrophy; cTnl, cardiac troponin l; DMD, Duchenne muscular dystrophy; IQR, interquartile range

*Values are statistically significant according to Mann-Whitney's $U$ test.

\$Values are statistically significant according to Fisher's exact test.

\section{Changes in serum cTnl levels, LVEF, and LVDd in the second decade of life}

As shown in Fig. 1, abnormal cTnl values were generally found in the second decade of life in patients with DMD and BMD. Since the onset of cardiomyopathy was generally observed in the second decade of life among DMD patients [27, 28], we hypothesized that the elevation of serum cTnl was an initial abnormal finding suggesting cardiac involvement. To evaluate this, annual changes in serum cTnl levels and echocardiographic findings were described (Fig. 2). Only the first measurement of serum cTnl, LVEF, or LVDd was adopted for each individual if the parameters were measured multiple times at the same age. In DMD patients, the median serum cTnl level was elevated to the abnormal range (cTnl $\geq 0.05$ $\mathrm{ng} / \mathrm{mL}$ ) at 11 years of age. It reached a maximum at 13 years, and thereafter, it decreased with age and returned to the normal range at 16 years of age (Fig. 2a). Median LVEF decreased with age and became abnormal (LVEF $<53 \%$ ) at the age of 14 years-a year after the maximum cTnl level was observed. Cardiac dysfunction (LVEF $<53 \%$ ) was observed three years after abnormal median serum cTnl levels (Fig. 2b). Median LVDd gradually increased but did not reach an abnormal value (LVDd > 55 mm; Fig. 2c). In BMD patients, serum cTnl value also increased with age and peaked at the age of 14 years. Thereafter, it decreased with age (Fig. 2d). In contrast to the finding in DMD patients, the median peak of serum cTnl 
$(0.04 \mathrm{ng} / \mathrm{mL})$ in BMD patients did not exceed the upper limit of the standard value $(\geq 0.05 \mathrm{ng} / \mathrm{mL})$. LVEF decreased with age and became abnormal at the age of 17 years (Fig. 2e)-three years after the age at which cTnl peaked in BMD patients. LVDd gradually increased but did not reach an abnormal value; this finding was similar to the observation among DMD patients (Fig. 2f).

\section{ACTN3 genotype and serum cTnl levels in patients with DMD}

We have previously reported that the ACTN3 XX null genotype is related to the early onset of dilated cardiomyopathy in DMD patients [26]. To evaluate the contribution of the ACTN3 genotype to cTnl level, patients with DMD were grouped into three genotypes: the RR, RX, and XX genotypes. As the ACTN3 genotype was analyzed only for patients whose genomes were conserved, the genotype was determined in 73 of the 127 DMD patients. Genotypes RR, RX, and XX were identified in 14 (19.18\%), 38 (52.05\%), and $21(28.77 \%)$ of the 73 patients, respectively. Patients with the ACTN3 XX genotype had maximum cTnI levels at the age of 11 years. On the other hand, in patients with RR and RX genotypes, cTnl peaked at the ages of 14 and 12 years, respectively (Fig. 3a). The highest median cTnI values were $0.16,0.21$, and 0.29 $\mathrm{ng} / \mathrm{mL}$ in the RR, RX, and XX groups, respectively. Patients with the XX genotype had a higher cTnl peak value at an earlier stage of disease than those with other genotypes. To determine the impact of alphaactinin-3 deficiency, patients were divided into two groups-XX group and RR and RX group-and we compared the annual change in cTnl levels between the two groups. The peak of $\mathrm{cTn}$ in the XX group occurred three years earlier than in the RR and RX group (Fig. 3b).

\section{Discussion}

We confirmed that higher cTnl levels were generally found in the second decade of life in patients with DMD and BMD. Second, the median serum cTnl level by age was higher in DMD patients until the age of 18 years, and abnormal cTnl values were more common in the DMD group than in the BMD group. In addition, the median maximum cTnl levels were found one year before the median abnormal LVEF value in DMD patients and three years before in BMD patients. Finally, we found that the ACTN3 XX genotype showed higher cTnl elevation earlier than the other two genotypes in DMD patients.

Cardiac dysfunction due to the progression of cardiomyopathy is the main cause of death in patients with DMD and BMD [29]. Therefore, international guidelines recommend early detection and therapy for cardiomyopathy in DMD and BMD patients [2,30]. There are, however, some difficulties associated with diagnosing cardiomyopathy since the age of onset and severity can be variable [30], and symptoms due to deterioration of cardiac function, such as orthopnea and dyspnea on exertion or rest, are rare in DMD patients [10]. Nigro et al. reported that $61.5 \%$ of patients with DMD had preclinical cardiomyopathy without any symptom [6]. Perloff et al. reported that even asymptomatic DMD patients showed regional wall motion abnormalities after 10 years of age [31]. Early detection of cardiomyopathy in patients with DMD may, therefore, be relevant because timely initiation of medications or therapies will delay cardiac remodeling and relieve cardiac dysfunction. Biomarkers that can detect the onset of cardiomyopathy are thus required, and our study suggests that cTnl may be such a biomarker. 
cTnl is a member of the troponin complex and a major component of myofibrils, and it is uniquely expressed in cardiac muscles [32]. It appears in the blood following cardiac injury and is a specific cardiac injury marker [15]. Its levels are elevated not only in acute but also in chronic pathogenic conditions. Recently, increasing cTnl levels have been shown in patients with cardiomyopathy or chronic $\mathrm{HF}$ in the general population $[33,34]$. In the present study, $55.9 \%$ and $31.9 \%$ of the patients with DMD and $B M D$, respectively, had elevated cTnl levels. Although Kan et al. reported that DMD patients who had acute cardiomyopathy with acute chest pain had elevated cTnl levels and diffuse ST changes on ECG [35], none of the patients in our study complained of symptoms related to acute myocardial injury such as chest pain or dyspnea. Moreover, echocardiography and ECG did not show any sign of AMI. These results, therefore, indicated that chronic myocardial injury caused elevation of cTnl levels in patients with DMD and BMD in our study.

In the present study, we found higher serum cTnl levels with increasing age in DMD patients compared with BMD patients, and the proportion of patients with abnormal serum cTnl levels was larger in the DMD group at all ages and in the age range of $10<$ years $\leq 18$ when compared with the BMD group. In one study, cardiomyopathy was ubiquitously observed in patients with DMD (in more than $90 \%$ of patients over 18 years of age) [7]. On the other hand, the onset of cardiomyopathy was variable in patients with BMD. The onset of dilated cardiomyopathy, the typical end form of cardiomyopathy, occurs in the midteen years to 20s in DMD patients [7, 36] and 30s to 40s in BMD patients [37]. These results indicate that DMD generally has an earlier and more severe cardiac phenotype than BMD. In fact, DMD patients exhibited more severe LVEF decline compared to BMD patients. This difference in severity can be explained by the difference in dystrophin levels in the myocardium. In comparison with BMD patients who have an in-frame mutation that produces shortened and less functional dystrophin, DMD patients have a complete absence of dystrophin, resulting in myocardial disruption by mechanical stress [38]. The difference in the level of cTnl between the two groups of patients is considered to reflect the different degrees of myocardial damage in the two muscular disorders.

Previous studies have reported the utility of $\mathrm{cTnl}$ for evaluating the cardiac function, especially in patients with DMD. Matsumura et al. reported that most DMD patients showed higher levels of cTnl in the second decade of their lives; however, no obvious correlation between cTnI and LVEF or brain natriuretic peptide was observed [39]. Hammere-Lercher et al. reported that all patients with DMD, with a mean age of 7.5 years, had cTnl levels below the upper reference limit (URL), and there was no relation of cTnl level to clinical evidence of cardiac failure [18]. Castro-Cago et al. also reported no relationship between cTnl levels and cardiac function [40]. These reports suggest that cTnl cannot be used to evaluate cardiac function. However, as shown in our study, the cTnl level was transiently elevated in the second decade before the decline of LVEF. This indicated that the cTnl level was not associated with cardiac function at the time of measurement, but later, it was.

Recently, myocardial fibrosis (MF) in DMD patients has been demonstrated using cMRI with late gadolinium enhancement (LGE), which revealed that subepicardial fibrosis was the main characteristic of DMD patients [41]. When the myocardium is injured, damaged cardiomyocytes are repaired by 
recruitment, proliferation, and activation of cardiac fibroblasts, which produce extracellular matrix components, resulting in the formation of fibrotic scars [42]. Remarkably, MF has been reported in DMD cardiomyopathy before the onset of myocardial dysfunction in young patients with DMD [43]. We hypothesize that serum cTnl levels may increase with the progression of MF because the observed timings of fibrosis and cTnl rise are the same (early second decade of life). Recently, Sonia et al. reported that cTnI values correlated with cMRI findings in patients with DMD cardiomyopathy [44]. They showed that cTnl levels in DMD patients with mild LGE were significantly increased compared to those in patients without LGE. These studies and the present study indicate that cTnl, a standard marker for AMI, may have the potential to become an alternative, cost-effective, and noninvasive biomarker for detecting early signs of cardiac injury. In fact, cTnl has gained popularity as a biomarker in the diagnosis of HF [45], and the cost of cTnl assay has been reported to be 10-100 times less than that of cardiac imaging [46].

As measurable plasma cTnl is found in the healthy population $[24,47]$, the abnormal value of $c T n l$ is recommended to exceed the 99th percentile URL [48]. However, there is no internationally accepted standard for the 99th percentile URL of cTnl, although a wide range of variables has been used as the 99th percentile URL [49]. Caselli et al. recently reported plasma cTnl levels in healthy neonates, children, and adolescents; 357 participants had a high sensitive immunoassay similar to that used in our study. In their study, the cTnl showed the highest value in the first weeks of life, and it decreased progressively up to adulthood. Therefore, the 99th percentile URL needs to be defined according to age. They reported that the 99th percentile URL was age dependent; it was $61.3 \mathrm{ng} / \mathrm{L}$ for the whole population minus neonates and infants $(1<$ years $\leq 18)$ and $41.3 \mathrm{ng} / \mathrm{L}$ for the group of adolescents $(10<$ years $\leq 18)$ [24]. Unfortunately, they did not report the 99 th percentile URL for toddlers $(1<$ years $\leq 10)$. Therefore, we decided to define the 99th percentile URL according to the patient's age as follows: $\geq 0.07 \mathrm{ng} / \mathrm{mL}, 1<$ years $\leq 10 ; \geq 0.05 \mathrm{ng} / \mathrm{mL}, 10<$ years $\leq 18$; and $\geq 0.03 \mathrm{ng} / \mathrm{mL}$, $>18$ years (manufacturer's recommendation).

In the present study, we also examined the relationship between the ACTN3 genotype and cTnl levels in patients with DMD and found that the maximum cTnl level in patients with ACTN3 XX genotype was observed a few years earlier compared with the other two genotypes. These results suggest that patients with the XX genotype may have a higher risk for myocardial injury. Interestingly, the existence of alphaactinin-3 has been reported not only in skeletal muscles but also in human fetal and adult hearts [50]. We recently reported that the $\mathrm{XX}$ genotype is related to a lower LV dilation-free survival rate in patients with DMD [22]. The impact of alpha-actinin-3 deficiency on cardiomyopathy progression was not elucidated in this study. Our results, however, indicate that alpha-actinin-3-deficient myocardium can be sensitive to mechanical and/or hypoxic damage that induces elevation of cTnl levels.

This study has some limitations. First, it was a retrospective observational study and was subject to selection bias. Second, although we evaluated a relatively large number of patients with DMD and BMD compared to previous studies, the number of participants may not be enough to allow generalization of our results to larger cohorts. However, the rarity of these muscular disorders may make it difficult to conduct studies on larger samples. Third, no patient had cTnl level measured over a long follow-up 
period; therefore, we could not elucidate precise changes in serum cTnl level for each patient by age. Finally, we did not assess the effects of cardioprotective medications that might affect cTnl levels. In addition, since we measured cTnl level only once at assessment for each patient, we do not know whether the value is reproducible or not. Despite these limitations, our study is unique in that it is a longitudinal study with a large number of DMD or BMD patients. Our findings may positively impact cardiac care by supporting the use of cTnl as a biomarker for cardiomyopathy.

In conclusion, we evaluated and compared serum cTnl levels and cardiac function in patients with DMD or BMD in a large cohort. cTnl levels were higher in DMD patients compared with BMD patients of each age group until the second decade of life, suggesting myocardial injury indicated was more severe in DMD patients. The ACTN3 null genotype may be a risk factor for early myocardial injury.

\section{Methods}

\section{Study design and subjects}

This retrospective, clinical observational study was conducted with the approval of the Ethics Committee of Kobe University (Approval No. 1534). Informed consent was obtained from the patients or their parents. The study protocol conformed to the ethical guidelines of the 1975 Declaration of Helsinki.

We reviewed the electronic charts of patients with DMD or BMD at Kobe University Hospital. Between August 1, 1991 and May 15, 2019, 459 DMD and 104 BMD patients were followed up. Of these, patients whose cTnI was measured at least once were enrolled in this study. The clinical diagnoses of DMD or BMD were confirmed by the identification of mutations in the $D M D$ gene. Gene mutations were analyzed in both genomic DNA and mRNA extracted from muscle or lymphocytes, as described previously [23].

\section{Serum cTnl measurements and reference values}

Serum cTnl levels were measured with Architect STAT cardiac troponin I assay (Abbott Diagnostics) until April 14, 2015. Thereafter, the assay method was changed to Architect STAT highly sensitive Tnl assay (Abbott Diagnostics) using the Architect $2000_{\mathrm{SR}}$ platform in our hospital. The limit of detection was $<10$ $\mathrm{pg} / \mathrm{mL}$ and $1.1-1.9 \mathrm{pg} / \mathrm{mL}$, and the 99th percentile values were 28 and $26 \mathrm{pg} / \mathrm{mL}$, respectively, for the former and latter assay methods, as reported by the manufacturer. When the acquired data was less than a minimum reference value, we defined the value as zero. Abnormal serum cTnl levels were defined as follows: $\geq 0.07 \mathrm{ng} / \mathrm{mL}$ (patient's age: $1<$ years $\leq 10$ ), $\geq 0.05 \mathrm{ng} / \mathrm{mL}$ (patient's age: $10<$ years $\leq 18$ ), and $\geq 0.03 \mathrm{ng} / \mathrm{mL}$ (patient's age: $>18$ years), according to previous literature and the manufacturer's recommendations [24, 25].

\section{Echocardiography}

A detailed echocardiogram method has been previously described [23]. Briefly, all 
echocardiograms were performed by T. Yamamoto. Echocardiographic evaluation of patients with DMD or BMD was scheduled annually until the age of 12 years and biannually thereafter. All patients were placed in the supine position during the investigation. Cardiac dysfunction was defined as left ventricular ejection fraction (LVEF) < 53\% [23]. LV dilation was defined as LV end-diastolic dimension (LVDd) $>55$ $\mathrm{mm}$ [23].

\section{ACTN3genotyping}

The ACTN3 genotype was determined only in DMD patients whose genomic DNA was conserved in our laboratory. Genomic DNA was isolated using standard phenol-chloroform extraction methods. ACTN3 exon 15 was amplified by polymerase chain reaction (PCR) as previously described [26]. The purified PCR-amplified products were sequenced using the Premix sequencing system (Fasmac Co., Ltd., Kanagawa, Japan). If patients had a variant in two alleles of c.1729C (p.577R), the ACTN3 genotype was defined as 577RR (RR). If patients had a single nucleotide variant of c.1729C > T (p. R577X) in one allele, the ACTN3 genotype was defined as 577RX (RX), and in two alleles, as 577XX (XX).

\section{Statistical analysis}

Data were expressed as numbers and percentages or medians and interquartile ranges (IQR). MannWhitney's U test or Fisher's exact test was used, as appropriate, for statistical analysis of the results. A difference was considered statistically significant when the $p$-value was $<0.05$. Analyses were performed using GraphPad Prism 5.0 (GraphPad Software, San Diego, CA, USA).

\section{Abbreviations}

ACTN3

alpha-actinin-3; AMI:acute myocardial infarction; BMD:Becker muscular dystrophy; cMRI:cardiovascular magnetic resonance imaging; CTnl:cardiac troponin I; DMD:Duchenne muscular dystrophy; ECG:electrocardiogram; HF:heart failure; IQR:interquartile range; LGE:late gadolinium enhancement; LV:left ventricular; LVEF:left ventricular ejection fraction; LVDd:left ventricular end-diastolic dimension; MF:myocardial fibrosis; PCR:polymerase chain reaction; URL:upper reference limit

\section{Declarations}

\section{Acknowledgments}

We thank the Clinical and Translational Research Center of Kobe University Hospital for the statistical analysis of the data.

\section{Competing interests}

The authors declare no competing interests.

\section{Author contributions}


H.Y.: Conceptualization, Methodology, Formal Analysis, Investigation, Writing-Original Draft. H.A.: Conceptualization, Methodology, Investigation, Writing-Review \& Editing, Supervision, Project Administration, Funding Acquisition. T.Y.: Resources, Writing-Review \& Editing, Supervision. M.M.: Writing-Review \& Editing, Supervision. K.I.: Writing-Review \& Editing, Supervision.

\section{References}

1. Nakamura, A. \& Takeda, S. Mammalian models of Duchenne muscular dystrophy: pathological characteristics and therapeutic applications. J. Biomed. Biotechnol. 2011, 184393; https://doi.org/10.1155/2011/184393 (2011).

2. Birnkrant, D. J. et al. Diagnosis and management of Duchenne muscular dystrophy, part 1: diagnosis, and neuromuscular, rehabilitation, endocrine, and gastrointestinal and nutritional management. Lancet. Neurol.17, 251-267 (2018).

3. Marty, B. et al. Comprehensive evaluation of structural and functional myocardial impairments in Becker muscular dystrophy using quantitative cardiac magnetic resonance imaging. Eur. Heart $J$. Cardiovasc. Imaging.20, 906-915 (2019).

4. Comi, G. P. et al. Clinical variability in Becker muscular dystrophy. Genetic, biochemical and immunohistochemical correlates. Brain.117, 1-14 (1994).

5. Falzarano, M. S., Scotton, C., Passarelli, C. \& Ferlini, A. Duchenne muscular dystrophy: from diagnosis to therapy. Molecules.20, 18168-18184 (2015).

6. Nigro, G., Comi, L. I., Politano, L. \& Bain, R. J. The incidence and evolution of cardiomyopathy in Duchenne muscular dystrophy. Int. J. Cardiol.26, 271-277 (1990).

7. Bushby, K. et al. Diagnosis and management of Duchenne muscular dystrophy, part 1: diagnosis, and pharmacological and psychosocial management. Lancet Neurol.9, 77-93 (2010).

8. Kamdar, F. \& Garry, D. J. Dystrophin-deficient cardiomyopathy. J. Am. Coll. Cardiol.67, 2533-2546 (2016).

9. Bach, J. R. \& Martinez, D. Duchenne muscular dystrophy: continuous noninvasive ventilatory support prolongs survival. Respir. Care.56, 744-750 (2011).

10. D'Amario, D. et al. A current approach to heart failure in Duchenne muscular dystrophy. Heart.103, 1770-1779 (2017).

11. Hor, K. N., Mah, M. L., Johnston, P., Cripe, T. P. \& Cripe, L. H. Advances in the diagnosis and management of cardiomyopathy in Duchenne muscular dystrophy. Neuromuscul. Disord.28, 711716 (2018).

12. Bushby, K. et al. Diagnosis and management of Duchenne muscular dystrophy, part 2: implementation of multidisciplinary care. Lancet Neurol.9, 177-189 (2010).

13. Jefferies, J. L. et al. Genetic predictors and remodeling of dilated cardiomyopathy in muscular dystrophy. Circulation.112, 2799-2804 (2005). 
14. Spurney, C. F. et al. Feasibility and reproducibility of echocardiographic measures in children with muscular dystrophies. J. Am. Soc. Echocardiogr.28, 999-1008 https://doi.org/10.1016/j.echo.2015.03.003 (2015).

15. Brunklaus, A. et al. The value of cardiac MRI versus echocardiography in the pre-operative assessment of patients with Duchenne muscular dystrophy. Eur. J. Paediatr. Neurol.19, 395-401 (2015).

16. Ciafaloni, E. et al. Delayed diagnosis in Duchenne muscular dystrophy: data from the Muscular Dystrophy Surveillance, Tracking, and Research Network (MD STARnet). J. Pediatr.155, 380-385 (2009).

17. Gaze, D. C. \& Collinson, P. O. Multiple molecular forms of circulating cardiac troponin: analytical and clinical significance. Ann. Clin. Biochem.45, 349-355 (2008).

18. Hammerer-Lercher, A. et al. Clinical and experimental results on cardiac troponin expression in Duchenne muscular dystrophy. Clin. Chem.47, 451-458 (2001).

19. Alpert, J. S., Thygesen, K., Antman, E. \& Bassand, J. P. Myocardial infarction redefined-a consensus document of the Joint European Society of Cardiology/American College of Cardiology Committee for the redefinition of myocardial infarction. J. Am. Coll. Cardiol.36, 959-969 (2000).

20. Beggs, A. H. et al. Cloning and characterization of two human skeletal muscle alpha-actinin genes located on chromosomes 1 and 11. J. Biol. Chem.267, 9281-9288 (1992).

21. Hogarth, M. W. et al. Analysis of the ACTN3 heterozygous genotype suggests that a-actinin-3 controls sarcomeric composition and muscle function in a dose-dependent fashion. Hum. Mol. Genet.25, 866-877 (2016).

22. Nariai, H. et al. Scalp EEG ictal gamma and beta activity during infantile spasms: evidence of focality. Epilepsia.58, 882-892 (2017).

23. Yamamoto, T. et al. Cardiac dysfunction in Duchenne muscular dystrophy is less frequent in patients with mutations in the dystrophin Dp116 coding region than in other regions. Circ. Genom. Precis. Med.11, e001782 (2018).

24. Caselli, C. et al. Plasma cardiac troponin I concentrations in healthy neonates, children and adolescents measured with a high sensitive immunoassay method: high sensitive troponin I in pediatric age. Clin. Chim. Acta.458, 68-71 (2016).

25. Koerbin, G. et al. Characterisation of a highly sensitive troponin I assay and its application to a cardio-healthy population. Clin. Chem. Lab. Med.50, 871-878 (2012).

26. Nagai, M. et al. The ACTN3 577XX null genotype is associated with low left ventricular dilation-free survival rate in patients with Duchenne muscular dystrophy. J. Card. Fail.26, 841-848 (2020).

27. Takeshima, Y. et al. Mutation spectrum of the dystrophin gene in 442 Duchenne/Becker muscular dystrophy cases from one Japanese referral center. J. Hum. Genet.55, 379-388 (2010).

28. Connuck, D. M. et al. Characteristics and outcomes of cardiomyopathy in children with Duchenne or Becker muscular dystrophy: a comparative study from the Pediatric Cardiomyopathy Registry. Am. Heart J.155, 998-1005 (2008). 
29. Biggar, W. D. Duchenne muscular dystrophy. Pediatr. Rev.27, 83-88 (2006).

30. Feingold, B. et al. Management of cardiac involvement associated with neuromuscular diseases: a scientific statement from the American Heart Association. Circulation.136, e200-e231 (2017).

31. Perloff, J. K., Henze, E. \& Schelbert, H. R. Alterations in regional myocardial metabolism, perfusion, and wall motion in Duchenne muscular dystrophy studied by radionuclide imaging. Circulation.69, 33-42 (1984).

32. Cummins, B., Auckland, M. L. \& Cummins, P. Cardiac-specific troponin-I radioimmunoassay in the diagnosis of acute myocardial infarction. Am. Heart J.113, 1333-1344 (1987).

33. NACB Writing Group. et al. National Academy of Clinical Biochemistry laboratory medicine practice guidelines: use of cardiac troponin and B-type natriuretic peptide or $\mathrm{N}$-terminal pro-B-type natriuretic peptide for etiologies other than acute coronary syndromes and heart failure. Clin. Chem.53, 20862096 (2007).

34. Giannoni, A., Giovannini, S. \& Clerico, A. Measurement of circulating concentrations of cardiac troponin I and T in healthy subjects: a tool for monitoring myocardial tissue renewal? Clin. Chem. Lab. Med.47, 1167-1177 (2009).

35. Hor, K. N. et al. Progression of Duchenne cardiomyopathy presenting with chest pain and troponin elevation. J. Neuromuscul. Dis.4, 307-314 (2017).

36. Barp, A. et al. Genetic modifiers of Duchenne muscular dystrophy and dilated cardiomyopathy. PLOS ONE.10, e0141240 (2015).

37. Hoogerwaard, E. M. et al. Evolution of cardiac abnormalities in Becker muscular dystrophy over a 13year period. J. Neurol.244, 657-663 (1997).

38. Petrof, B. J., Shrager, J. B., Stedman, H. H., Kelly, A. M. \& Sweeney, H. L. Dystrophin protects the sarcolemma from stresses developed during muscle contraction. Proc. Natl. Acad. Sci. U. S. A. 90: 3710-3714(1993).

39. Matsumura, T., Saito, T., Fujimura, H. \& Shinno, S. Cardiac troponin I for accurate evaluation of cardiac status in myopathic patients. Brain Dev.29, 496-501 (2007).

40. Castro-Gago, M., Gómez-Lado, C. \& Eirís-Puñal, J. Cardiac troponin I for accurate evaluation of cardiac status in myopathic patients. Brain Dev.31, 184 (2009).

41. Puchalski, M. D. et al. Late gadolinium enhancement: precursor to cardiomyopathy in Duchenne muscular dystrophy? Int. J. Cardiovasc. Imaging.25, 57-63 (2009).

42. Pinto, A. R. et al. Revisiting cardiac cellular composition. Circ. Res.118, 400-409 (2016).

43. Silva, M. C. et al. Myocardial fibrosis progression in Duchenne and Becker muscular dystrophy: a randomized clinical trial. JAMA Cardiol.2, 190-199 (2017).

44. Voleti, S., Olivieri, L., Hamann, K., Gordish-Dressman, H. \& Spurney, C. Troponin I levels correlate with cardiac MR LGE and native T1 values in Duchenne muscular dystrophy cardiomyopathy and identify early disease progression. Pediatr. Cardiol.41, 1173-1179 (2020). 
45. Zhou, F. J. et al. Diagnostic value of analysis of H-FABP, NT-proBNP, and cTnl in heart function in children with congenital heart disease and pneumonia. Eur. Rev. Med. Pharmacol. Sci.18, 1513-1516 (2014).

46. Andreassi, M. G. et al. Imaging and laboratory biomarkers in cardiovascular disease. Curr. Pharm. Des.15, 1131-1141 (2009).

47. Caselli, C. et al. Distribution of circulating cardiac biomarkers in healthy children: from birth through adulthood. Biomark Med.10, 357-365 (2016).

48. Apple, F. S. et al. National Academy of Clinical Biochemistry and IFCC Committee for Standardization of Markers of Cardiac Damage Laboratory Medicine Practice Guidelines: analytical issues for biochemical markers of acute coronary syndromes. Clin Chem.53, 547-551 (2007).

49. Sandoval, Y. \& Apple, F. S. The global need to define normality: the 99th percentile value of cardiac troponin. Clin. Chem.60, 455-462 (2014).

50. Kim, M. S. et al. A draft map of the human proteome. Nature.509, 575-581 (2014).

\section{Figures}
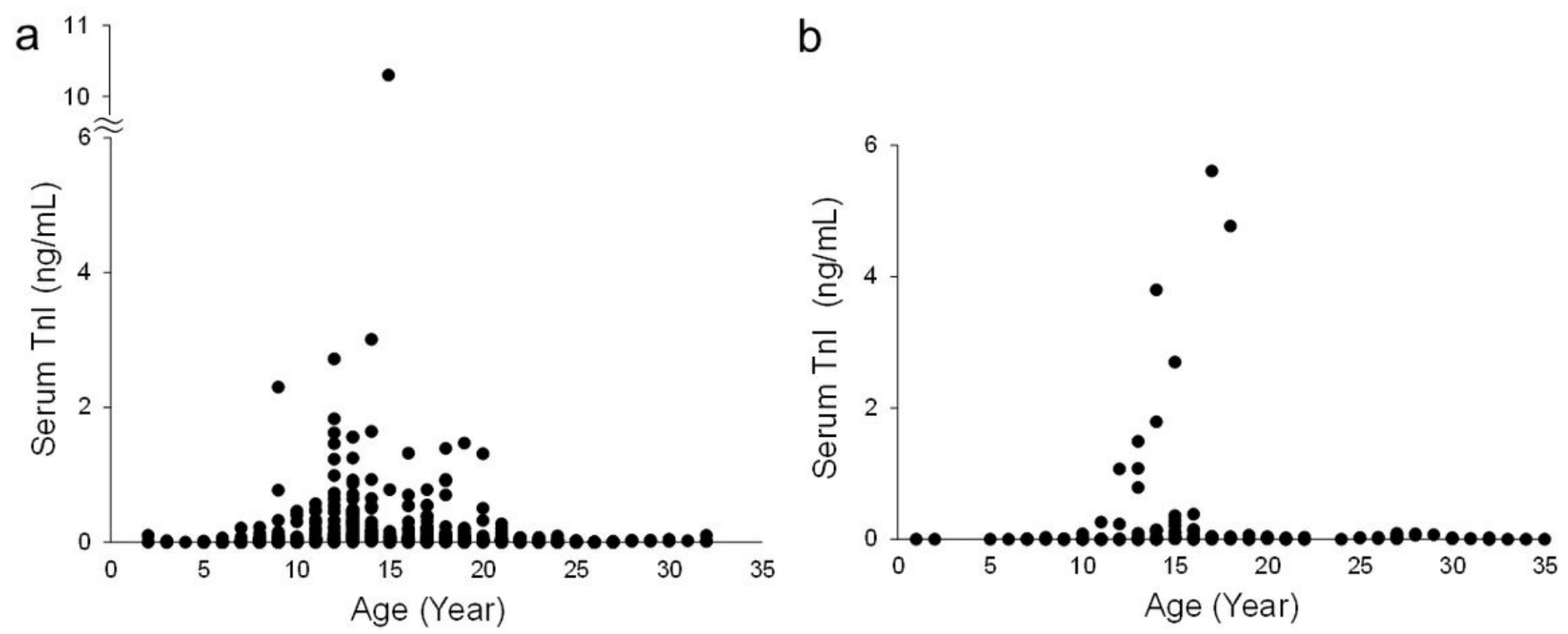

\section{Figure 1}

Serum cTnl levels in patients with DMD (a) and BMD (b) by age In all, 529 and 131 serum cTnl values were obtained from $127 \mathrm{DMD}$ and $47 \mathrm{BMD}$ patients, respectively. cTnl, cardiac troponin l; BMD, Becker muscular dystrophy; DMD, Duchenne muscular dystrophy 

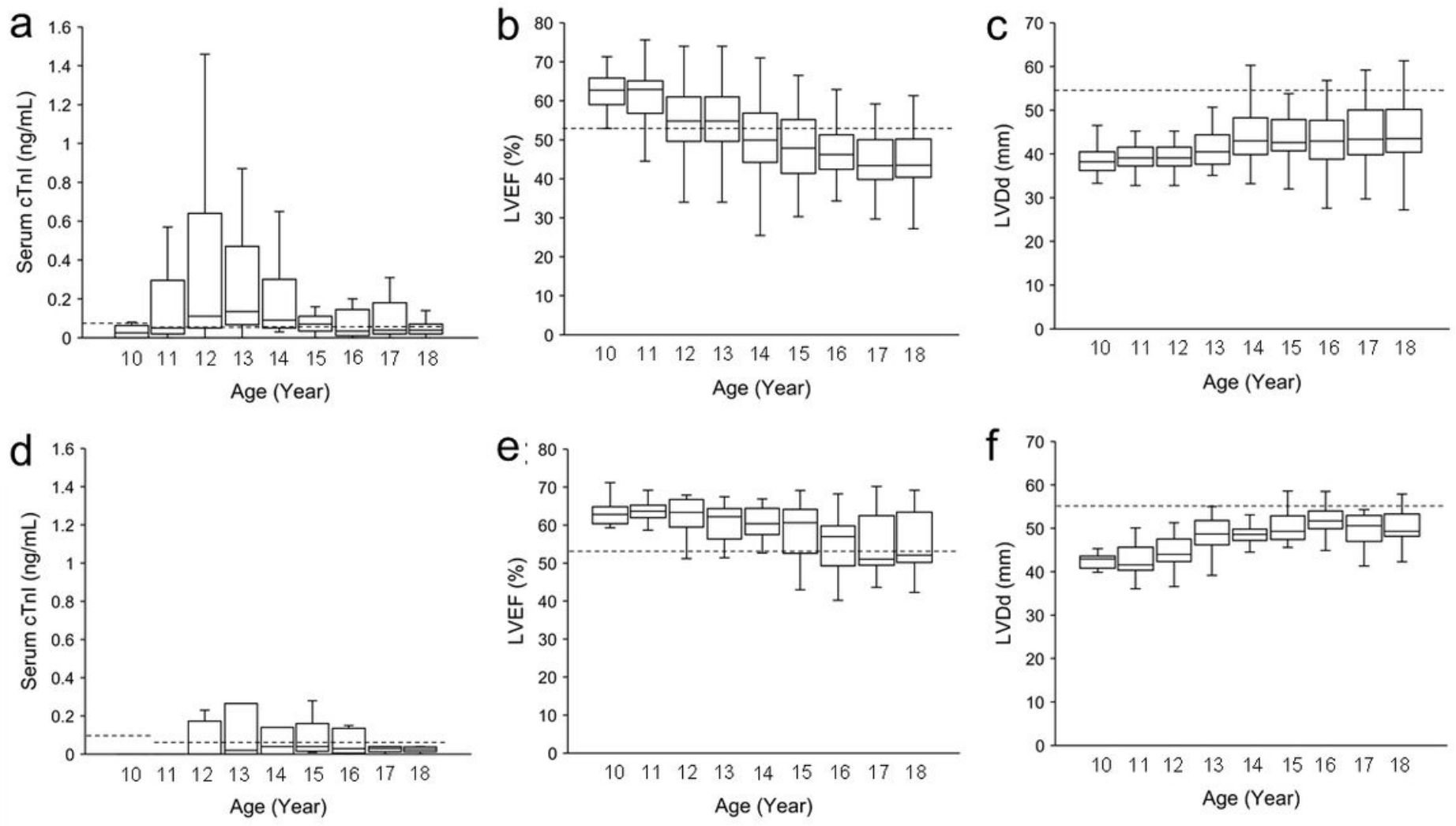

Figure 2

Serum cTnl levels, LVEF, and LVDd in the second decade of life in patients with DMD and BMD Dotted lines in a and $d$ show upper limits of reference values. Dotted lines in $b$ and $e$, and $C$ and $F$ indicate the assessment of cardiac dysfunction (LVEF $<53 \%$ ) and left ventricular dilation (LVDd $>55 \mathrm{~mm}$ ), respectively. A box and whisker plot show the first quartile to the third quartile. A vertical line goes through the box at the median. The upper and lower whiskers represent scores outside the middle $50 \%$. cTnl, cardiac troponin I; BMD, Becker muscular dystrophy; DMD, Duchenne muscular dystrophy; LVEF, left ventricular ejection fraction; LVDd, left ventricular end-diastolic dimension 

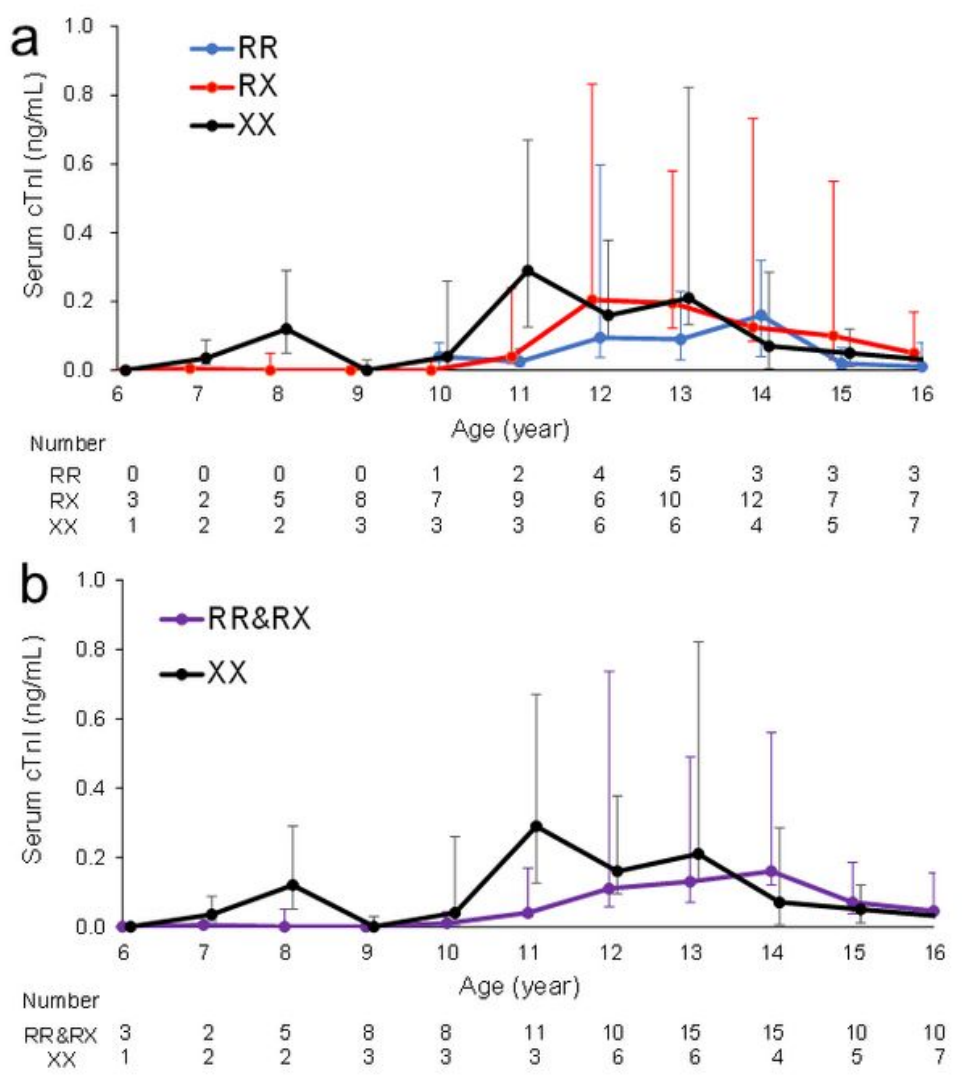

\section{Figure 3}

Changes in serum cTnl levels in patients with different ACTN3 genotypes The XX genotype showed an early onset of cTnl elevation (a). When compared between the XX genotype group and the RR and RX genotype groups, serum cTnl in the XX group peaked three years earlier (b). Data are represented as median, the positive vertical bar represents 75 th percentile, and the negative vertical bar represents 25 th percentile. RR, RX, and XX represent the ACTN3 577RR, 577RX, and 577XX genotypes, respectively. cTnl, cardiac troponin I 